\title{
1 Coronavirus RNA synthesis takes place within membrane-bound sites
}

2 Nicole Doyle ${ }^{1}$, Jennifer Simpson ${ }^{1}$, Philippa C Hawes ${ }^{1}$ and Helena J Maier ${ }^{1 *}$

$4{ }^{1}$ The Pirbright Institute, Ash Road, Woking, Surrey, GU24 ONF, UK; nicole.doyle@pirbright.ac.uk

5 (N.D.); jennifer.simpson@pirbright.ac.uk (J.S.); pippa.hawes@pirbright.ac.uk (P.C.H.)

6 * Correspondence: helena.maier@pirbright.ac.uk (H.J.M)

7

\section{Abstract}

Infectious bronchitis virus (IBV), a gammacoronavirus, is an economically important virus to the poultry industry as well as a significant welfare issue for chickens. As for all positive strand RNA viruses, IBV infection causes rearrangements of the host cell intracellular membranes to form replication organelles. Replication organelle formation is a highly conserved and vital step in the viral life cycle. Here, we investigate the localization of viral RNA synthesis and the link with replication organelles in host cells. We have shown that sites of viral RNA synthesis and virus-related dsRNA are associated with one another and, significantly, that they are located within a membrane-bound compartment within the cell. We have also shown that some viral RNA produced early in infection remains within these membranes throughout infection. Importantly, we demonstrate conservation across all four coronavirus genera, including SARS-CoV-2. Under-standing more about the replication of these viruses is imperative in order to effectively find ways to control them.

\section{Introduction}

Coronaviruses (CoVs) are an important family of positive strand RNA (+RNA) viruses with a wide host range. In humans, some strains of these viruses such as the human coronavirus (HCoV) 229E can cause the common cold, however there are now three CoVs that are more pathogenic and cause higher fatality rates. Until 2019, severe acute respiratory syndrome coronavirus (SARS-CoV) and Middle East respiratory syndrome coronavirus (MERS-CoV), which were initially isolated in China and Saudi Arabia, respectively [1, 2] were the most well-known zoonotic viruses in the coronavirus family. However, the emergence of SARS-CoV-2, causing global mortalities (over 4 million people at the time of writing [3]) and shut-down of normal life has brought a focus on the danger of zoonotic viruses, and in particular this virus family [4]. Apart from human viruses, coronaviruses also cause disease in a range of animal species. Several of these viruses are of economic importance as they cause infections and loss of income in the global agriculture industry. These include viruses such as porcine epidemic diarrhea virus (PEDV), porcine deltacoronavirus (PDCoV), bovine coronavirus (BCoV) and avian infectious bronchitis virus (IBV). As has recently been highlighted, studying these viruses is of vital importance. Much work on the CoV family over recent years has been focused on understanding how they interact with the host cell, including a key part of their life cycle, the induction of membrane rearrangements or replication organelles (ROs). 
As obligate intracellular parasites, viruses rely on their host cells to provide not only a site for replication, but much of the cellular machinery required to produce new virus particles. All + RNA viruses induce the rearrangement of intracellular membranes to form ROs [5-7]. These viral ROs have been shown to be the site of viral RNA synthesis [8, 9], however it is probable that they also offer other benefits to the virus. The ROs are likely to provide a way to shield replicative intermediates which would otherwise be recognized by cellular defenses and spark an innate immune response $[10,11]$. It has also recently been shown that ROs could be a site of local ATP production for the energy-intensive process of RNA synthesis which takes place there [12]. The structures formed vary between virus families but do share similarities in structures such as convoluted membranes, double membrane vesicles (DMVs) and spherules [13, 14]. Several viruses, such as toroviruses [15], hepatitis C virus (HCV; [16]) and picornaviruses such as foot and mouth disease virus (FMDV; [17]) induce the formation of tubules or paired membranes as well as single-membrane vesicles, DMVs or multi-lamellar vesicles. In enterovirus infections single-membrane tubules transform into DMVs and multilamellar vesicles over the course of infection [18, 19], using endoplasmic reticulum (ER) and Golgi membranes to initiate the formation of these structures [20]. A different structure induced by many other +RNA viruses are spherules or invaginated vesicles. These structures are smaller than DMVs and are pinched out from various intracellular membranes but because they remain bound to that membrane, they possess a channel which connects their interior to the cytoplasm. Viruses that induce spherule formation include flaviviruses [21-23], nodaviruses [24], bromoviruses [25] and alphaviruses such as Semliki Forest virus (SFV). In the case of SFV ROs, the spherules and the cytopathic vacuoles which contain them have been shown to be the site of viral RNA synthesis [26-28]. For flock house nodavirus (FHV), spherules have been found to form from the mitochondrial membrane [29] and these structures have also been shown to be the site of viral RNA synthesis, containing bundles of dsRNA [30, 31].

The possibility of antiviral therapies targeting the ROs has meant an increased attraction for understanding this part of the viral life cycle in recent years. Until very recently, the structures of ROs formed by the CoV family were split into the alpha- and beta-CoVs and the gamma- and delta-CoVs. The alpha- and beta-CoVs were known to induce convoluted membranes and DMVs [9, 32-35], and while DMVs are also produced by gamma- and delta-CoVs, these were seen alongside areas of tightly paired ER membranes called zippered ER (zER) and small double membrane spherules which, in IBV infected cells, have been shown to remain tethered via an open neck to the zER [36, 37]. However, it has recently been shown that alpha- and beta-CoVs also induce spherules, although with some morphological differences. They are formed to a lesser extent, and while some spherules appear to be connected to the convoluted membrane, many more were seen as sealed structures. While some sealed spherule structures were seen in IBV infection in the later study, this was to a lesser extent [8]. The significance of these differences between structures is not yet clear and is the source of further investigation.

As ROs have long been purported to provide a site for viral RNA synthesis, a key point for investigation has been in understanding exactly where the sites of viral replication are 
83 within the cell, and the involvement of ROs in the process. For SARS-CoV, it has been 84 shown using biochemical methods in vitro that sites of viral RNA synthesis are protected by membranes, possibly within DMVs [38], and dsRNA signal was shown to be associated with DMVs and CM using immunostaining [39]. SARS-CoV-2 DMVs have also recently been shown to contain RNA filaments consistent with the size of dsRNA [40]. It is assumed that DMVs in mouse coronavirus (MHV) play a role in viral RNA synthesis based on the fact that mutations in one of the non-structural proteins, nsp4, affects both DMV formation and viral RNA synthesis [41, 42]. DMVs were known to be necessary for viral replication [43] and while DMVs have long been associated with sites of viral RNA synthesis [9], this theory was always hampered by the fact that DMVs were shown to be closed compartments with no way for newly synthesized viral RNA to escape for packaging and egress. However, DMVs have now been shown to be a site of viral RNA synthesis [8] and further recent findings demonstrated a small number of transient pores in the membranes of MHV DMVs, allowing egress of newly synthesized viral RNA [44]. The role of spherules remains to be elucidated.

The gammacoronavirus, IBV is a virus of economic importance to the global poultry industry, causing a highly contagious respiratory disease of chickens and other poultry. Infection results in reduced quantity of eggs and reduced quality of eggs and meat as well as impacting on animal welfare. Recent work has elucidated the role of ROs in viral RNA synthesis and suggested a pathway for nascent RNA to leave the DMV via a molecular pore [44]. However, conclusive proof of viral RNA synthesis occurring within the DMV was still lacking and the possibility remained that synthesis was taking place on the outer surface of DMVs. Here, we show that IBV viral RNA synthesis takes place within membrane-bound compartments, and we demonstrate that this is conserved across all four genera of the CoV family.

\section{Materials and Methods}

\section{Cells and viruses}

Avian DF1 cells (LGC Standards Ltd.) were maintained in DMEM (Sigma Aldrich, Gillingham, UK) supplemented with 10\% FBS (Sigma Aldrich) at $37^{\circ} \mathrm{C} 5 \% \mathrm{CO}_{2}$. IBV (strain BeauR [45]) infections were carried out in 1XBES medium (MEM, 0.3\% tryptose phosphate broth, $0.2 \%$ bovine serum albumin, $20 \mathrm{mM} \mathrm{N}, \mathrm{N}$-Bis(2-hydroxyethyl)-2-aminoethanesulfonic acid (BES), $0.21 \%$ sodium bicarbonate, $2 \mathrm{mM} \mathrm{L}$-glutamine, $250 \mathrm{U} / \mathrm{mL}$ nystatin, $100 \mathrm{U} / \mathrm{mL}$ penicillin, and $100 \mathrm{U} / \mathrm{mL}$ streptomycin). Huh7 cells (ATCC) were maintained in DMEM supplemented with 10\% FBS. Human coronavirus 229E (HCoV 299E (UK Health Security Agency) infections were carried out in DMEM $+2 \%$ FBS. VeroE6 cells (LGC Standards Ltd.) were maintained in DMEM supplemented with 10\% FBS. SARS-CoV-2 (strain hCov-19/England/02/2020, kindly provided by Prof Miles Carroll, UK Health Security Agency) infections were carried out in DMEM $+2 \%$ FBS. Porcine LLC-PK1 cells (ATCC CL-101 [46]) were maintained in DMEM supplemented with 10\% FBS. PDCoV (strain OH-FD22, kindly provided by Prof Linda Saif, Ohio State University, [47, 48]), infection was carried out in EMEM + 1\% HEPES, $1 \%$ non-essential amino acids, $1 \%$ antibiotic/antimycotic and $0.25 \mu \mathrm{g} / \mathrm{mL}$ trypsin. In all cases, cells were inoculated with virus for $1 \mathrm{~h}$, after which time 
125 the inoculum was replaced with infection media specific to each virus. Cells were then

126 incubated until specified timepoints.

\section{Labeling of nascent viral RNA with bromouridine}

129 Cells seeded onto glass coverslips were infected as in 2.1. Cells were then treated with 2 $130 \mathrm{mM}$ (or $4 \mathrm{mM}$ for HCoV 229E) bromouridine (BrU; Sigma Aldrich) and $15 \mu \mathrm{M}$ actinomycin D 131 (ActD; Sigma Aldrich) at 30 min prior to each timepoint. Cells were washed in PBS, fixed in 132 RNase-free paraformaldehyde (pfm) at each timepoint, then labeled as in 2.3.

133 For pulse chase experiments, cells were pulsed with BrU and ActD for $1 \mathrm{~h}$ at concentrations 134 previously used. After this time, control cells were fixed in RNase-free pfm, while the rest 135 were chased with $50 \mu \mathrm{M}$ uridine (Sigma Aldrich) to out-compete the labeled BrU. Cells were 136 incubated in uridine and ActD until $24 \mathrm{hpi}$ then fixed as above and labeled as in 2.3.

139 Cells seeded onto glass coverslips were mock-infected or infected as laid out in 2.1. and 2.2.

140 At each timepoint, cells were washed in PBS then fixed for $15 \mathrm{~min}$ in $4 \% \mathrm{pfm}$ in PBS at room 141 temperature. Cells were permeabilized in $0.1 \%$ Triton X-100 in PBS for $15 \mathrm{~min}$, or $0.25 \%$ 142 digitonin (BioVision Inc) in PBS for 10 min then incubated in blocking buffer $(0.1 \%$ fish 143 gelatin [Sigma Aldrich] in PBS) for $1 \mathrm{~h}$. Primary antibodies specific for proteins of interest 144 (Table 1) were diluted in blocking buffer and incubated on cells for $1 \mathrm{~h}$. After washing, Alexa 145 Fluor secondary antibody (Invitrogen) in blocking buffer was incubated on cells for $1 \mathrm{~h}$, 146 followed by washing, labeling of nuclei using 4',6-diamidino-2-phenylindole (DAPI; Sigma 147 Aldrich) or ToPro3 (ThermoFisher) and mounting onto glass slides with Vectashield (Vector 148 Labs, Peterborough).

149 For labeling of BrU samples, in order to prevent loss of the BrU signal, immunofluorescence 150 (IF) labeling was promptly carried out in an RNase-free environment in the presence of $151 \mathrm{RNasin}$ at $0.133 \mathrm{U} / \mathrm{ml}$ (Promega, Southampton; [49]).

152 Cells were visualized using a Leica CLSM SP5, SP8 or Stellaris 5 microscope (Leica 153 Microsystems, Milton Keynes, UK). Super-resolution (stimulated emission depletion 154 (STED)) microscopy was performed using a Leica TCS SP8 STED 3X microscope with 155 inverted stand. STED Images were deconvolved using Huygens Professional software 15618.10 (Scientific Volume Imaging, Netherlands). Figures were assembled using Adobe 157 Photoshop.

158

Table 1. Table showing antibodies used in this paper

\begin{tabular}{ccc}
\hline Antibody name in text & Antibody target & Source \\
\hline dsRNA & J2 dsRNA & Scicons (English and Scientific \\
Nsp12 & IBV RdRp (nsp12) & $\begin{array}{c}\text { Maier et al mBio '13 } \\
\text { IBVing }\end{array}$ \\
IBV & IBV structural proteins (S, & Abcam $~ N$ ) \\
PDI & PDI & Enzo \\
b-tubulin & b-tubulin & ThermoFisher \\
BrU & Anti-Bromodeoxyuridine & Roche, Sigma \\
229E N & HCoV 229E N protein & MRC PPU Reagents and Services \\
SARS2 N & SARS-CoV2 N protein & MRC PPU Reagents and Services \\
PDCoV N & PDCoV NP antigen & 2B Scientific \\
\hline
\end{tabular}




\section{Results}

$162 D s R N A$ is found in membrane-bound compartments.

163 We have shown previously over the course of the IBV life cycle that nsp12, the viral 164 RNA-dependent RNA polymerase (RdRp), does not colocalize with dsRNA [37]. The role of

165 dsRNA is still a source of interest, but it is most likely to be a replicative intermediate, formed 166 during the replication of the viral genome. Interestingly, dsRNA has recently been shown to 167 be largely negative-sense viral RNA [50]. It has been shown in SARS-CoV that dsRNA is 168 found within DMVs, which are quite likely to store it, hidden from detection by intracellular 169 pattern recognition receptors [39]. The formation of dsRNA is a well conserved step across

$170+$ RNA virus families so we sought to understand whether dsRNA is also protected within 171 membrane-bound compartments in IBV infection using different permeabilization agents.

172 Digitonin is a weak permeabilizing agent, which can be used at low concentrations to 173 selectively permeabilize the plasma membrane but not intracellular membranes, while Triton

174 X-100 (TX100) permeabilizes all cellular membranes. Mock-infected DF1 cells were 175 permeabilized with TX100 (Fig 1, top) or digitonin (Fig 1, bottom), then labeled with 176 antibodies specific for PDI and tubulin. Tubulin proteins are found in the cytoplasm, so 177 tubulin labeling was visible upon either TX100 or digitonin permeabilization. In contrast, 178 labeling of PDI, the intra-lumenal ER protein is lost with digitonin treatment as the antibody is 179 prevented from accessing its target. DF1 cells infected with IBV and fixed at timepoints 180 during the infection cycle as indicated (Fig 1) were similarly processed. On the top row, cells 181 permeabilized with TX100 clearly show both nsp12 (green) and dsRNA (red) labeling, with 182 the dsRNA labeling increasing markedly over the course of infection. However, when cells 183 were permeabilized with digitonin (Fig 1, bottom row), dsRNA was no longer visible in the 184 cells, indicating that it is held within an intracellular membrane and therefore inaccessible to 185 the antibody. In comparison, nsp12 staining was unaffected when cells were permeabilized 186 with digitonin (Fig 1), indicating that it is not contained within a membrane but is possibly free 187 in the cytoplasm or on the cytoplasmic face of a membrane. 


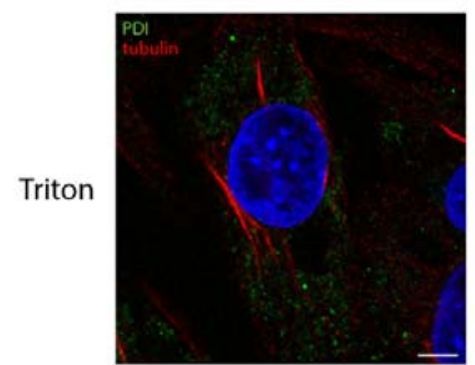

Digitonin

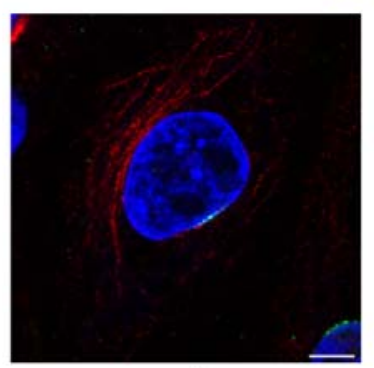

mock
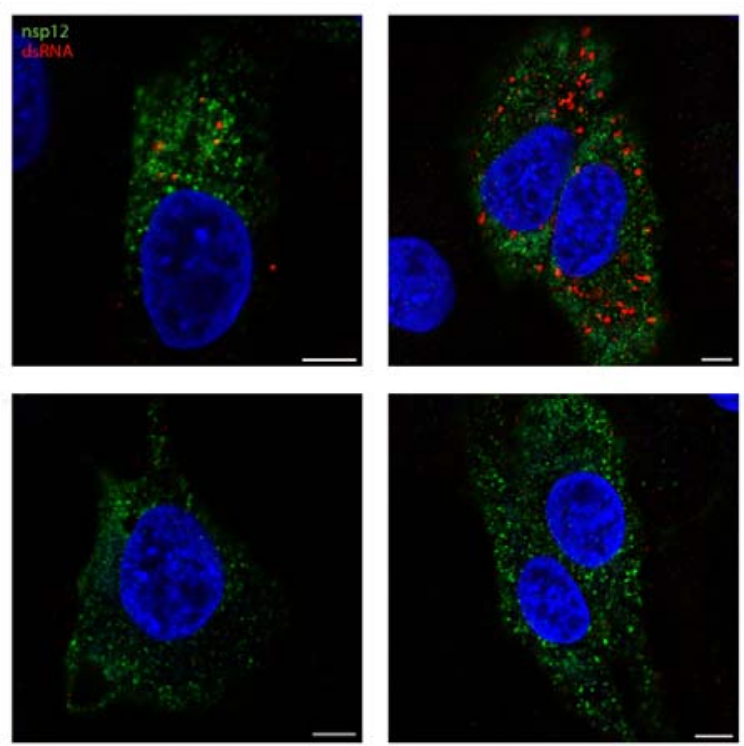

$6 \mathrm{hpi}$

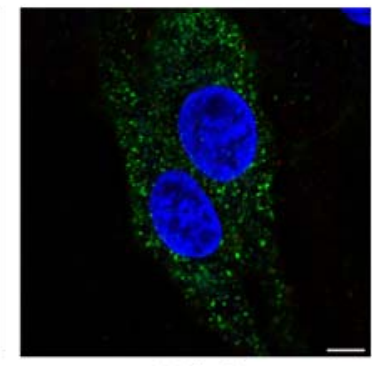

$10 \mathrm{hpi}$
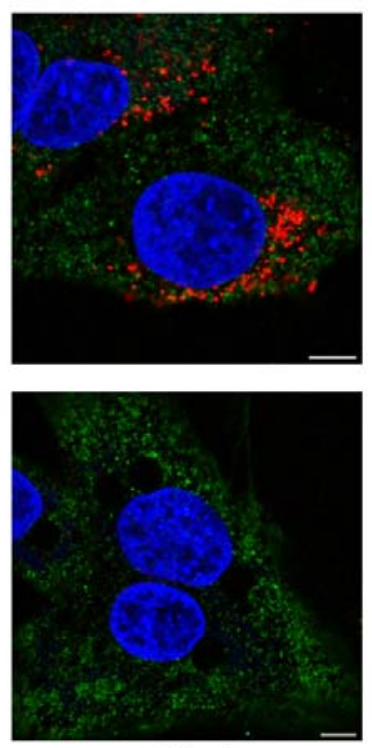

$24 \mathrm{hpi}$

Figure 1. DsRNA is contained within a membrane-bound compartment whilst nsp12 is exposed to the cytoplasm.

DF1 cells were infected with IBV and fixed at the indicated times post infection. Cells were permeabilized with either Triton X-100 (all membranes permeabilized; top row), or digitonin (plasma membrane permeabilized only; bottom row). Cells were labeled with dsRNA (red) and nsp12 (green), or for the mock control (first column), tubulin (red) and PDI (green). Nuclei are labeled blue with DAPI (blue). Scale bars represent $5 \mu \mathrm{m}$.

DsRNA is closely associated with sites of viral RNA synthesis.

Knowing that dsRNA was likely within the DMVs, we sought to understand how it associated with sites of viral RNA synthesis. To do this, the uridine analog bromouridine (BrU) was incorporated into the nascent viral RNA. DF1 cells were infected with IBV then incubated in media containing $\mathrm{BrU}$ for 30 min prior to fixation to provide a snapshot of RNA synthesis at that timepoint. The cellular transcription inhibitor, Actinomycin D (ActD) was used to selectively inhibit cellular transcription to allow visualization of sites of active viral RNA synthesis (Fig S1). Starting at 4 hpi, sites of viral RNA synthesis (Fig 2, green) were detected as small foci localized in the perinuclear region. As infection progressed, these sites of viral RNA synthesis increased in size and distribution around the cell, although more often than not retaining their perinuclear distribution. A similar pattern was observed for both nsp12 and dsRNA labeling (red), however while nsp12 labeling was generally diffuse over the cytoplasm, dsRNA labeling remained punctate and tended to accumulate in perinuclear regions, especially earlier in infection (Fig 2b). Over the course of infection with IBV, nsp12 did not colocalize with BrU (Fig 2a). This was surprising as we would expect the viral RdRp to be at sites of viral RNA synthesis, however no colocalization could be found even when analyzed in 3D (Fig S2 and Video S3) or using super resolution microscopy (Fig 2c). In 
216 contrast, dsRNA, appeared to exhibit a low level of colocalization or overlap with BrU signal 217 at earlier timepoints, particularly in larger foci and as infection progressed, the overlap 218 appeared to increase (Fig 2b). Using super-resolution microscopy, we confirmed that rather 219 than tightly colocalizing, dsRNA and BrU signal were instead in close association with each 220 other (Fig 2c). These results show that dsRNA, while not appearing to be completely 221 colocalized with BrU-labeled nascent RNA, is closely associated with these sites of viral 222 RNA synthesis. 
bioRxiv preprint doi: https://doi.org/10.1101/2021.11.04.467246; this version posted November 4, 2021. The copyright holder for this preprint (which was not certified by peer review) is the author/funder, who has granted bioRxiv a license to display the preprint in perpetuity. It is made available under aCC-BY-ND 4.0 International license.

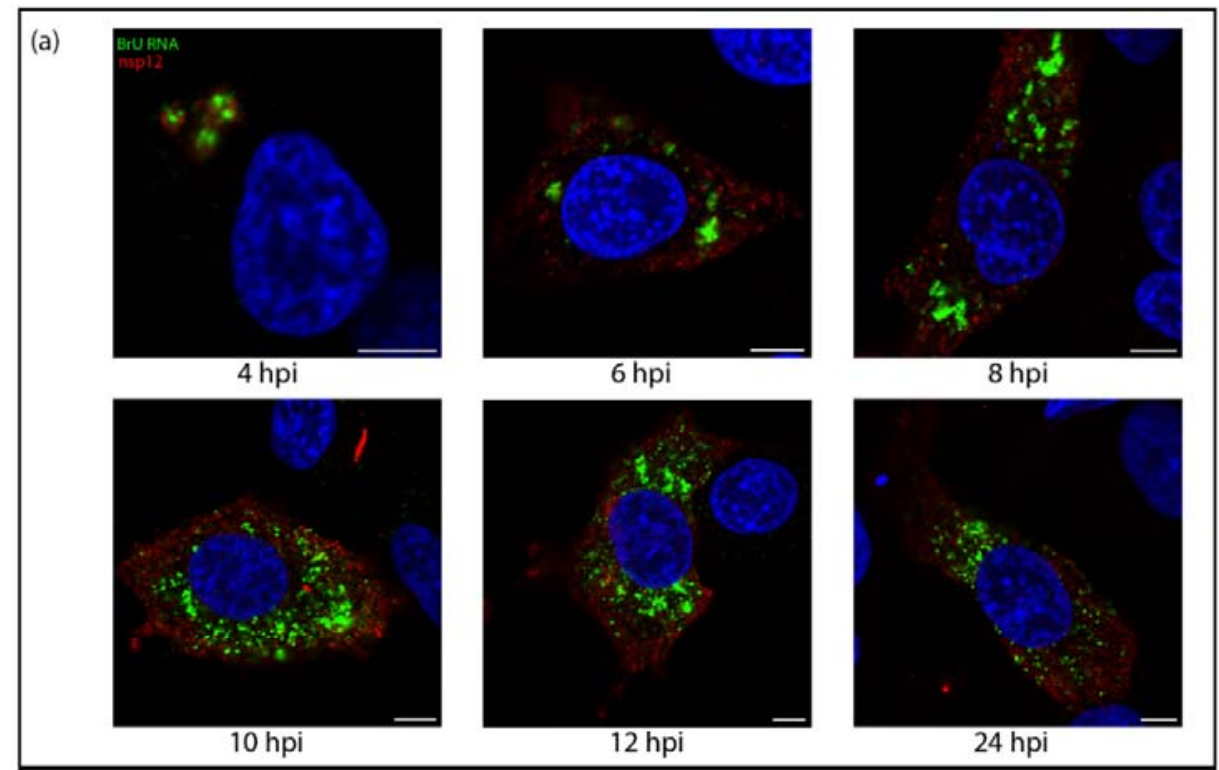

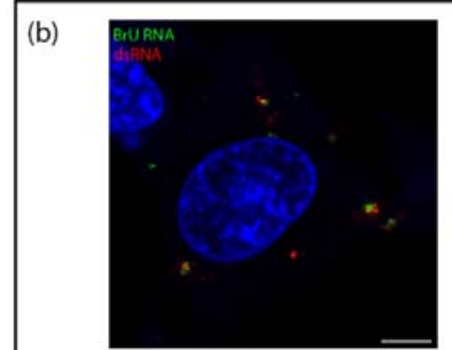

$4 \mathrm{hpi}$

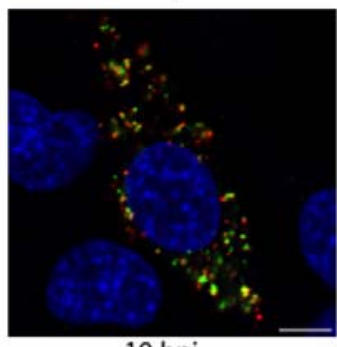

$10 \mathrm{hpi}$

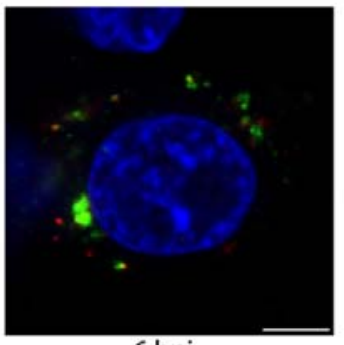

$6 \mathrm{hpi}$

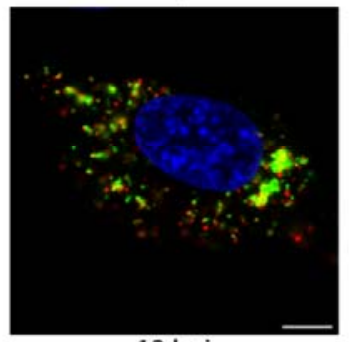

$12 \mathrm{hpi}$

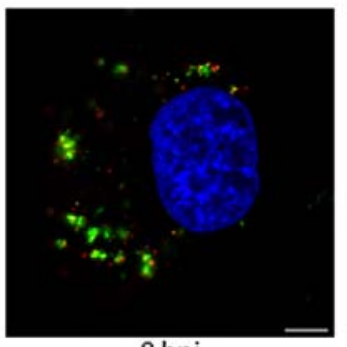

$8 \mathrm{hpi}$

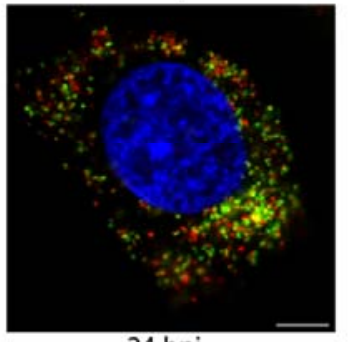

$24 \mathrm{hpi}$

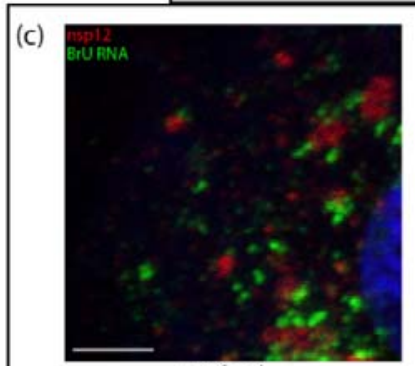

confocal

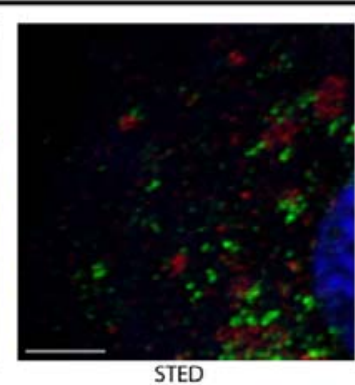

STED

(d)

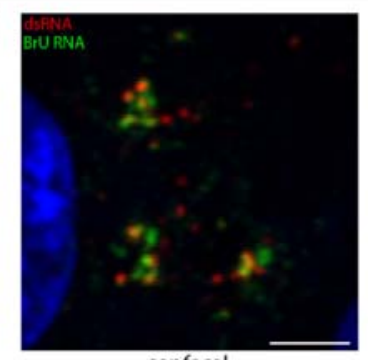

confocal

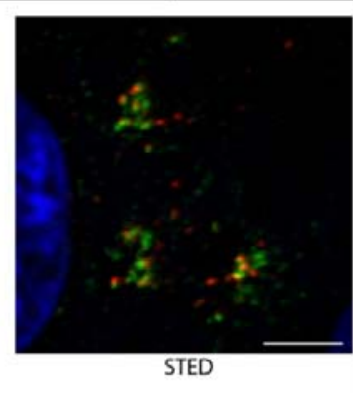

Figure 2. Sites of viral RNA synthesis are associated with dsRNA but do not colocalize with nsp12.

DF1 cells were infected with IBV and 30 mins prior to fixation treated with BrU and ActD. Cells were fixed at the indicated time points post infection and labeled with antibodies against $\mathrm{BrU}$ (green) and a) nsp12 (red) or b) dsRNA (red). Nuclei are labeled with DAPI (blue). Scale bars represent $5 \mu \mathrm{m}$. (c \& d) Cells were treated as in ( $\mathrm{a} \& \mathrm{~b}$ ), fixed at $10 \mathrm{hpi}$ and labeled for $\mathrm{BrU}$ (green) and c) nsp12 or d) dsRNA 
(red) and ToPro3 (blue) for the nuclei. A confocal image was captured followed by a super-resolution images which was captured using a STED microscope, then deconvolved. Scale bars represent $3 \mu \mathrm{m}$.

\section{Sites of viral RNA synthesis are membrane-protected.}

Snijder et al [8] showed that sites of viral RNA synthesis are associated strongly with ROs, in particular they showed they were more strongly associated with DMVs. While they subsequently showed that DMVs have a pore which would allow for the egress of newly synthesized viral RNA, the question of pinpointing nascent viral RNA within these structures remained [44]. Here it has been demonstrated that sites of IBV viral RNA synthesis and dsRNA are closely associated rather than precisely colocalized. Since we had already shown that dsRNA was within membrane-bound compartments, this left the question open as to whether the sites of viral RNA synthesis might be on the outside of these vesicles. DF1 cells were infected and treated with $\mathrm{BrU}$ and $\mathrm{ActD}$ as before, followed by IF labeling using either TX100 or digitonin permeabilization. As in previous experiments, the labeling pattern of newly synthesized viral RNA in TX100-permeabilized cells increased through the course of infection from smaller puncta to larger foci, mostly centered in perinuclear regions (Fig 3 , top row). Through the course of infection, nsp12 signal was unaffected by digitonin permeabilization (Fig 3 and as before, in Fig 1). Strikingly however, much of the $\mathrm{BrU}$ signal at these timepoints was not detectable following digitonin permeabilization (Fig 3, bottom row). Although some newly synthesized viral RNA was found in the cytoplasm, this observation indicates that a large proportion of newly synthesized viral RNA is bound within a membrane. Overall viral RNA labeling followed a very similar staining pattern to dsRNA (as seen in Fig 1) and suggests that nascent viral RNA is located within the same membrane-bound compartments as dsRNA. 

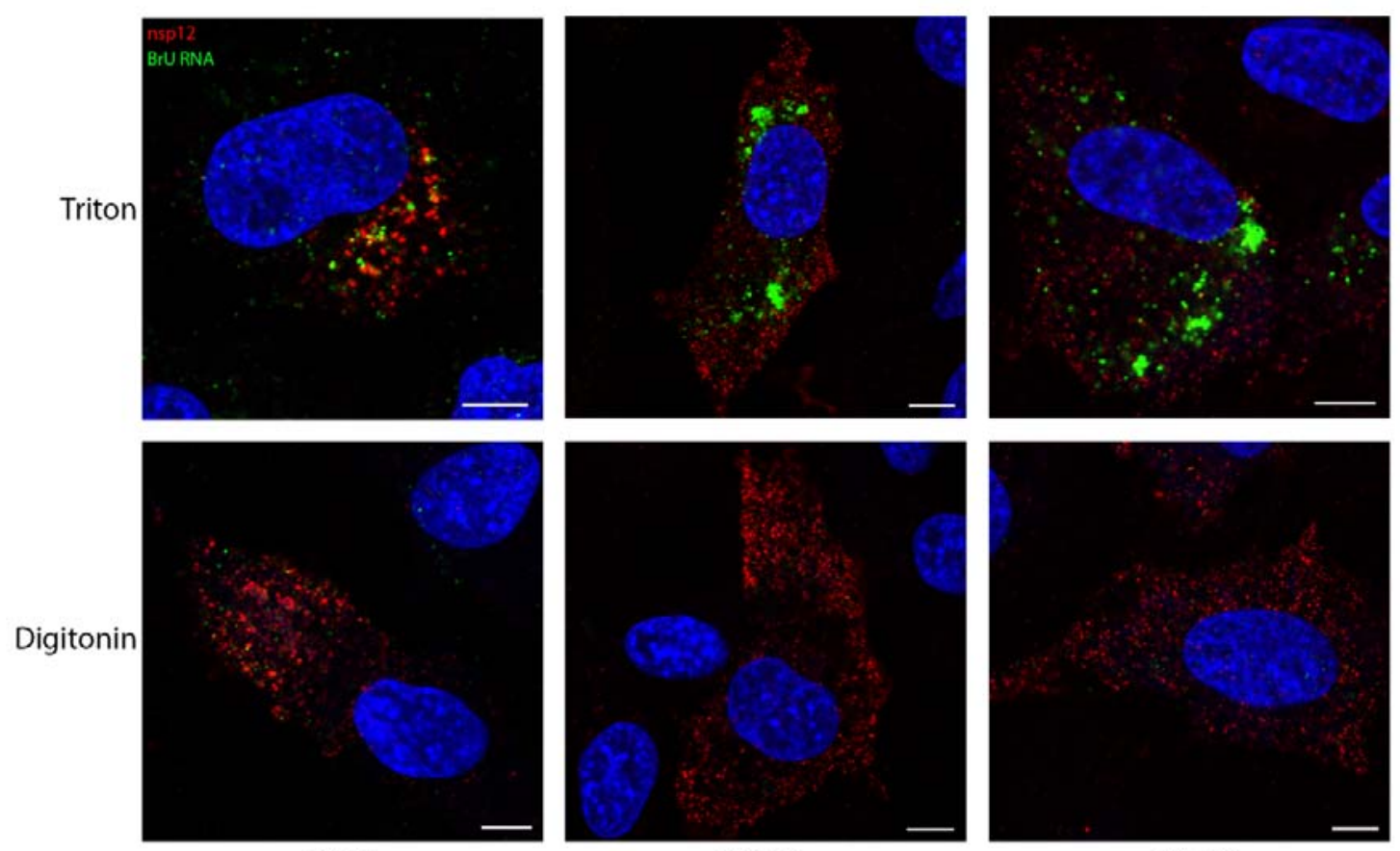

6 hpi

10 hpi

$24 \mathrm{hpi}$

Figure 3. Viral RNA synthesis takes place in a membrane-bound compartment.

DF1 cells were infected with IBV. 30 mins prior to fixation, cells were treated with BrU and ActD. Cells were fixed at the indicated times post infection. Cells were permeabilized with Triton X-100 (all membranes; top row) or digitonin (plasma membrane; bottom row) then labeled for nsp12 (red) and $\mathrm{BrU}$ (green), nuclei labeled with DAPI (blue). Scale bars represent $5 \mu \mathrm{m}$.

\section{Viral RNA is transported to the cytoplasm later in infection.}

To trace the fate of viral RNA synthesized early in infection and find out whether viral RNA produced within membrane-bound compartments is transported to the cytoplasm, cells were successively exposed to labeled uridine in the form of bromouridine (pulse) and then to unlabeled uridine (chase). In these pulse-chase experiments, viral RNA produced between 7-8 hpi was labeled with BrU as before, followed by a chase with excess unlabeled uridine until fixation at $24 \mathrm{hpi}$. The localization of viral RNA at $8 \mathrm{hpi}$ was in cytoplasmic puncta, consistent with earlier observations (Fig 4a, left). When this viral RNA was chased to $24 \mathrm{hpi}$, it was found localized in large cytoplasmic puncta but also diffuse within the cytoplasm. (Fig 4a, right). To gain further information, cells were permeabilized with TX100 or digitonin as before. In control cells fixed at $8 \mathrm{hpi}$ and permeabilized with TX100 or digitonin, labeling of the viral RNA was as before (Fig 4b, left). In the pulse-chased samples, the large foci of BrU-labeled RNA were no longer visible. However, the diffuse cytoplasmic signal was still detected (Fig 4b, right). Therefore, one pool of RNA was located within a membrane-bound compartment and a second was free in the cytoplasm. To investigate whether the cytoplasmic viral RNA might be in the process of being packaged, we looked to confirm that it colocalizes with viral structural proteins. Therefore, cells were labeled with an antibody specific for viral structural proteins (spike, membrane and nucleocapsid proteins). The 
bioRxiv preprint doi: https://doi.org/10.1101/2021.11.04.467246; this version posted November 4, 2021. The copyright holder for this preprint (which was not certified by peer review) is the author/funder, who has granted bioRxiv a license to display the preprint in perpetuity. It is made available under aCC-BY-ND 4.0 International license.

279 colocalization between these markers (Fig 4c, top) indicates that the diffuse cytoplasmic 280 staining pattern of BrU-labeled viral RNA is associated with structural proteins. In contrast, 281 dsRNA was shown to colocalize with the large membrane-bound foci (Fig 4c, bottom). 282

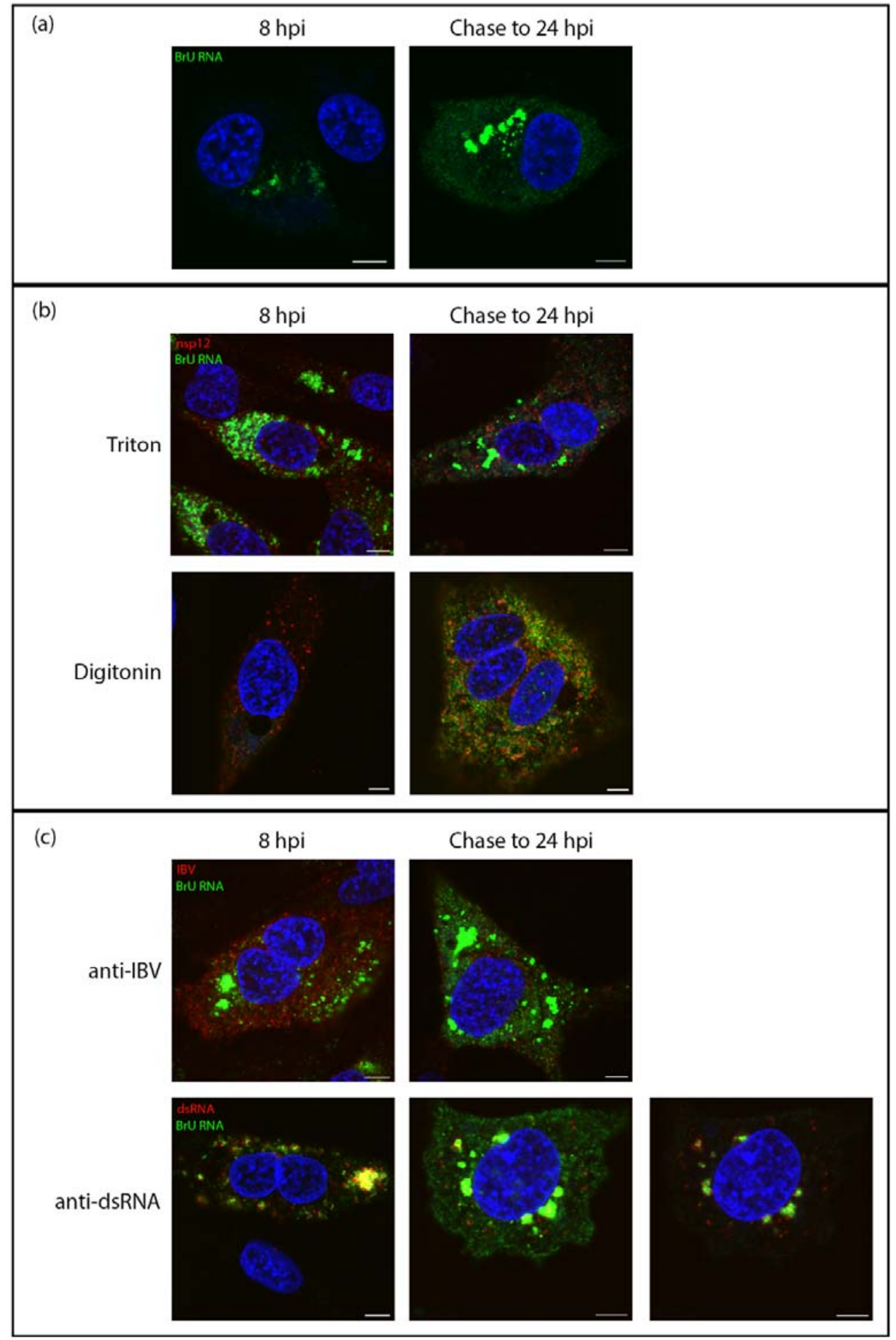


DF1 cells were infected with IBV. At 7 hpi cells were treated with BrU and ActD. At 8 hpi cells were either fixed (8 hpi) or chased with uridine until $24 \mathrm{hpi}$ (chase to $24 \mathrm{hpi}$ ). Cells were labeled for BrU (green) and (b) nsp12, (c) IBV or (d) dsRNA (red), DAPI labeling nuclei (blue). Scale bars represent 5 $\mu \mathrm{m}$.

\section{RNA synthesis by all genera of coronaviruses takes place in a membrane-bound} compartment.

While the structure of ROs has been shown to be conserved across all genera of CoVs, some morphological differences between the viruses do remain. Mainly, while convoluted membranes are found much more widely in alpha and beta CoV, they are found to a lesser extent in delta- and gamma-CoVs. In alpha- and beta-CoVs, the spherules were found associated with $\mathrm{CM}$ rather than $\mathrm{zER}$ and the majority of spherules were sealed compartments rather than remaining open to the cytosol as is the case in the majority of spherules in gamma-CoV infections [8]. We therefore sought to understand whether there might be any fundamental differences between the localization of sites of viral RNA synthesis in IBV compared with other CoV genera. Using one representative virus from each of the three other genera of CoVs (HCoV 229E (an alpha-CoV), SARS-CoV-2 (a beta-CoV) and PDCoV (a delta-CoV)), the location of nascent viral RNA labeled with BrU was assessed using TX100 and digitonin as before. Cells were labeled to detect BrU labeled nascent viral RNA or the viral nucleocapsid $(\mathrm{N})$. While for each virus the $\mathrm{N}$ labeling (in red) was detected diffuse throughout the cytoplasm regardless of permeabilization method, BrU-labeled nascent viral RNA signal (in green) was contained within a membrane-bound compartment for HCoV 229E (Fig 5a), SARS-CoV-2 (Fig 5b) and PDCoV (Fig 5c). This is consistent with observations for IBV and demonstrates a conserved mechanism across the CoV family for viral RNA synthesis to be held within a membrane-bound compartment. 

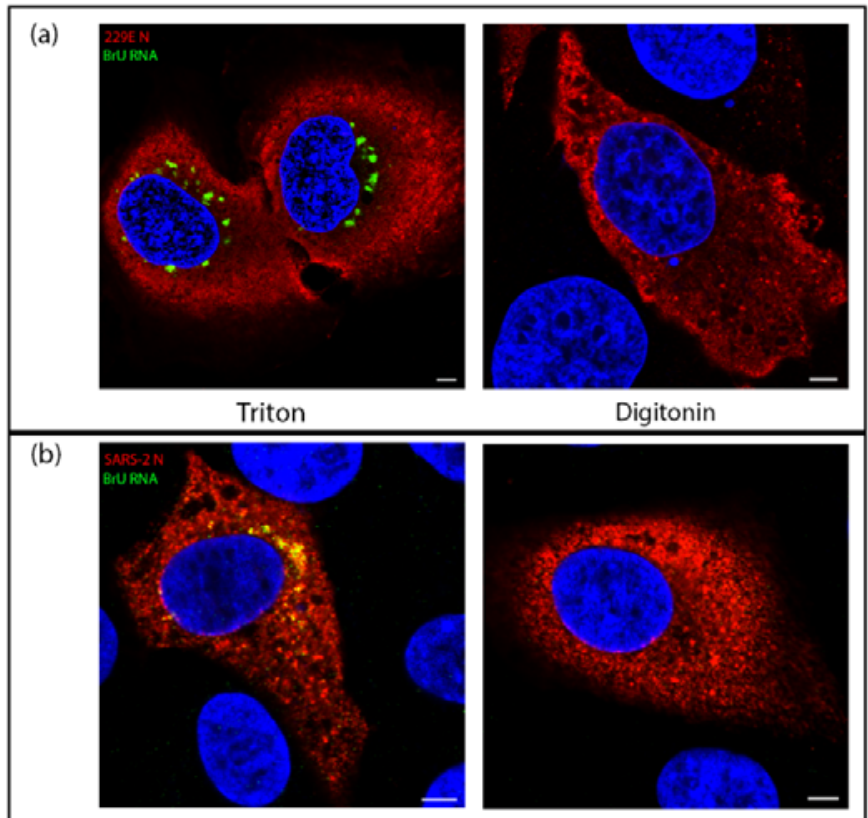

Triton
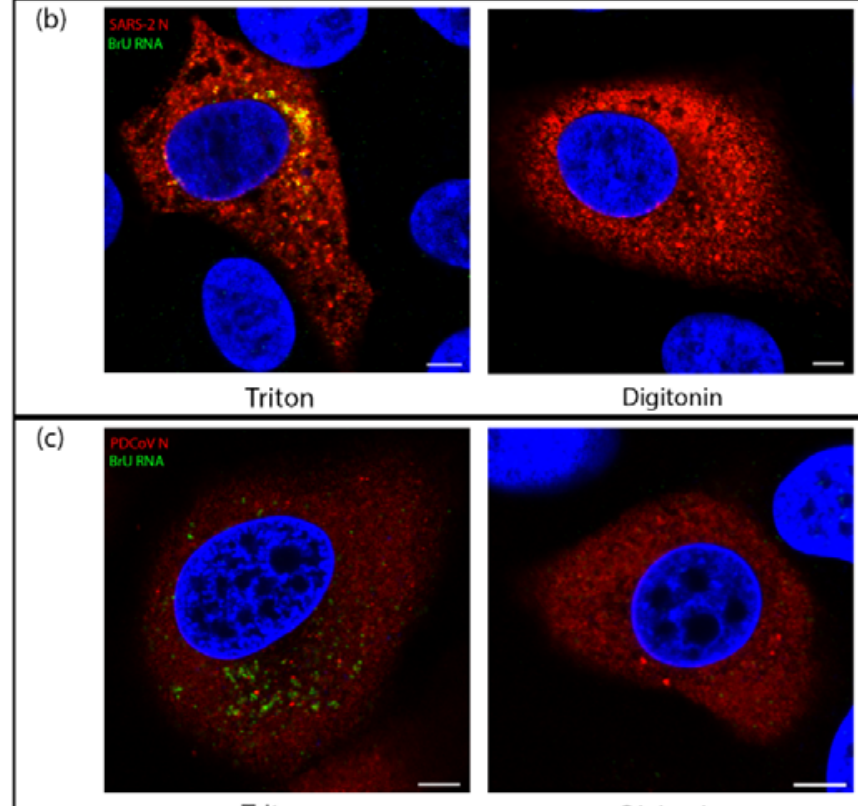

Digitonin

Figure 5. Viral RNA synthesis of diverse CoVs takes place within a membrane-bound compartment.

(a) Huh7 cells were infected with HCoV 229E. At 7.5 hpi cells were treated with BrU and ActD for 30 min before fixation at 8 hpi. Cells were permeabilized with Triton X-100 (left) or digitonin (right) then labeled for BrU (green) and $\mathrm{N}$ (red), DAPI labeling the nuclei (blue). (b) VeroE6 cells were infected with SARS-CoV-2. At $5.5 \mathrm{hpi}$ cells were treated with $\mathrm{BrU}$ and ActD for $30 \mathrm{~min}$ before fixation at $6 \mathrm{hpi}$. Cells were permeabilized with Triton $\mathrm{X}-100$ (left) or digitonin (right) then labeled for $\mathrm{BrU}$ (green) and $\mathrm{N}$ (red), DAPI labeling the nuclei (blue). (c) LLC-PK1 cells were infected with PDCoV. At 5.5 hpi cells were treated with $\mathrm{BrU}$ and $\mathrm{ActD}$ for $30 \mathrm{~min}$ before fixation at 6 hpi. Cells were permeabilized with Triton X-100 (left) or digitonin (right) then labeled for BrU (green) and N (red), DAPI labeling the nuclei (blue). Scale bars represent $5 \mu \mathrm{m}$.

\section{Discussion}

325 The formation of ROs is conserved across all +RNA viruses, an essential step in their life 326 cycle. These ROs have long been thought to provide a site for viral RNA synthesis and in 327 fact have been shown to do so for several viruses [8, 30, 31]. While these structures do vary 328 between the different virus families, there are many similarities, the most basic of them being 329 the energy required to rearrange cellular membranes to this extent. As obligate intracellular 
330 parasites, viruses are highly efficient and aim entirely to produce new generations of 331 infectious particles as soon as possible. That these viruses induce these structures at all 332 indicates that they are an important step in their life cycle [41, 43]. The gammacoronavirus, 333 IBV induces the formation of DMVs, zER and spherules which pinch out from, but remain 334 tethered to the $\mathrm{ZER}$. While IBV DMVs were recently identified as a site for viral RNA 335 synthesis, no role has yet been found for spherules [8].

336 Previous studies have shown for other +RNA viruses such as SARS-CoV, FHV, and 337 most recently in SARS-CoV-2 that dsRNA is found associated with DMVs [30, 31, 39, 40]. In 338 the current study we have shown using different permeabilization methods, that dsRNA is 339 found located within membrane-bound compartments. This is consistent with previous work 340 [39] and suggests that dsRNA is also shielded within DMVs during IBV infection. Although a 341 pore has recently been shown to be present in the DMV membrane [44], these are short 342 lived/transient and have a diameter of 2-3nm at their narrowest point, which would not be 343 large enough to allow entry of an antibody complex of $\sim 30 \mathrm{~nm}$. It is worth noting however, 344 that our data here cannot exclude the possibility of dsRNA association with spherules. The 345 IBV spherule neck measured 4-5 nm [37], which would also not be large enough to allow 346 entry of an antibody complex. As dsRNA is a known target for intracellular pattern 347 recognition receptors, it is likely that the virus aims to shield the dsRNA from detection within 348 membrane-bound compartments such as DMVs. Indeed, activation of interferon (IFN) 349 signaling is delayed following IBV infection and it was suggested that the IFN response then 350 seen later in IBV infection could be due to dsRNA "leaking" from DMVs [51]. However, this 351 now does not seem to be the case as data presented in the current work demonstrated that 352 dsRNA was sealed within a membrane compartment at $24 \mathrm{hpi}$, a timepoint after which IFN 353 signaling has been activated. Therefore, other mechanisms must exist to allow activation of 354 IFN signaling later in IBV infection and these remain to be elucidated.

$355 \quad$ Finding sites of coronavirus RNA synthesis has been a topic of much research in recent 356 years. Using biochemical methods in vitro, it has been shown that SARS-CoV RNA 357 synthesis takes place inside membrane-bound compartments [38]. More recently, it was 358 shown that DMVs are the site of viral RNA synthesis during MERS-CoV and IBV replication 359 [8]. However, the methods used in that study could not definitively show whether RNA 360 synthesis takes place on the interior or cytoplasmic face of DMV membranes. A subsequent 361 study showed that DMVs contain pores within the membrane connecting the interior of the 362 vesicle with the cytoplasm [44], providing a route by which RNA synthesized inside DMVs 363 could exit for translation or packaging. Despite this, the location of viral RNA synthesis either 364 inside or on the outside of DMVs remained to be confirmed. Significantly, here, by employing 365 both BrU labeling of nascent RNA and different permeabilization methods, we have shown 366 that sites of coronavirus RNA synthesis are membrane protected. Therefore, we have 367 demonstrated conclusively that viral RNA synthesis in fact takes place within a membrane 368 membrane-bound compartment. Moreover, we have confirmed that viral RNA synthesis by 369 diverse CoVs from each of the four coronavirus genera, including recently identified 370 SARS-CoV-2, takes place within a membrane-bound compartment. Although we cannot 371 exclude that CoV RNA synthesis takes place associated with spherules, as MERS-CoV and 
372 IBV RNA synthesis has been shown to be associated with DMVs [8], we can infer here that

373 RNA synthesis takes place within DMVs, and that this is conserved across the whole CoV 374 family.

375 While it has previously been shown that newly synthesized IBV RNA location overlaps

376 to some extent with the nsp14 [52], little else is known about the localization of viral proteins

377 to sites of viral RNA synthesis. Several viral nsps are known to be involved in RO formation,

378 including nsp3, nsp4 and nsp6 [39, 41, 43, 53-55] and nsps are known to localize to DMVs

379 and other RO membranes [34, 39, 56]. Here, we have shown that viral RNA is associated

380 with dsRNA but not with nsp12. While it was not surprising that as a replicative intermediate,

381 dsRNA was found in close association with sites of viral RNA synthesis, the finding that

382 nsp12 is not near these sites is somewhat surprising. However, this is likely to be because

383 the antibody is unable to bind to nsp12 assembled within the replication/transcription

384 complex (RTC) and actively involved in RNA synthesis. Nsp8 has been shown to interact

385 with both the $\mathrm{N}$ - and $\mathrm{C}$-terminal ends of nsp12 [57] which could very likely render the

386 antigenic sites within nsp12 inaccessible to something as large as an antibody. Nsp12 also

387 interacts directly with other viral proteins. MHV nsp12 interacts with nsp15 [58] and possibly

388 with nsp5, nsp8 and nsp9 [59]. SARS-CoV nsp12 has been found in complex with nsp7,

389 nsp8 and nsp14 [60]. Therefore, it is very likely that the nsp12 labeling detected here

390 represents nsp12 that is not located within RTCs or is located within RTCs not actively

391 involved in RNA synthesis. The amount of nsp12 signal detected that is presumably not

392 located within active RTCs is perhaps rather surprising. However, several nsps from other

393 CoVs are known to localize to both DMVs and other RO membranes [34, 39, 56]. The role of

394 non-RTC associated nsp12 during virus replication remains to be determined.

395 Following characterization of the location of nascent RNA throughout infection, changes

396 in the location of this RNA as infection progressed were studied. Using a pulse-chase

397 approach, RNA synthesized between 7-8 hpi was visualized at $24 \mathrm{hpi}$. This demonstrated

398 that by $24 \mathrm{hpi}$, viral RNA that was produced between 7-8 hpi showed two separate pools.

399 The first remained in large, membrane-bound foci. These large accumulations of labeled

400 viral RNA continued to associate with dsRNA within these compartments. The second pool

401 of viral RNA produced between 7-8 hpi and tracked to $24 \mathrm{hpi}$ had been exported into the

402 cytoplasm. This pool of RNA is associated largely with structural proteins, presumably

403 bound by $\mathrm{N}$, or as it was being packaged into new virions and is consistent with the recently

404 characterized pore in the DMV membrane [44]. These observations are consistent with a

405 model whereby newly synthesized positive sense RNAs are exported out of the DMV to be

406 translated or packaged, while positive and negative sense RNA templates remain within the

407 DMVs for further rounds of RNA synthesis.

408 The CoV family contains many pathogens of animal and human interest. The RO of 409 CoVs from all four genera have been confirmed previously to comprise DMVs and double 410 membrane spherules. Here we investigated the site of viral RNA synthesis of one virus from 411 each CoV genus. In all viruses we investigated (HCoV 229E, SARS-CoV-2, IBV and 412 PDCoV) the site of viral RNA synthesis was bound within a membrane, consistent with being 413 located on the interior of DMVs [8,44]. The role of CoV induced convoluted membranes, 
414 zippered ER and double membrane spherules remains elusive. However, knowing that all 415 CoVs synthesize viral RNA within a membrane-bound compartment is a significant step in 416 understanding the replication of this important virus family.

Supplementary Materials: The following are available online at www.mdpi.com/xxx/s1, Figure S1: Cellular transcription is inhibited with Actinomycin D treatment, Figure S2: Sites of viral RNA synthesis are associated with dsRNA but do not colocalize with nsp12., Video S3: Sites of viral RNA synthesis do not colocalize with nsp12; Video S4: Sites of viral RNA synthesis are associated with dsRNA.

Author Contributions: Conceptualization, H.J.M. and P.C.H. methodology, N.D., J.S., P.C.H. and H.J.M.; validation, N.D.; formal analysis, N.D.; investigation, N.D.; resources, N.D., P.C.H. and H.J.M.; data curation, N.D.; writing-original draft preparation, N.D. and H.J.M.; writing-review and editing, N.D., J.S., P.C.H., and H.J.M.; visualization, N.D.; supervision, H.J.M.; project administration, H.J.M.; funding acquisition, H.J.M. All authors have read and agreed to the published version of the manuscript.

Funding: This research was funded by Biotechnology and Biological Sciences Research Council, grant numbers BB/N002350/1, BBS/E///00002535, $\mathrm{BBS} / \mathrm{E} / \mathrm{I} / 00007034, \mathrm{BBS} / \mathrm{E} / \mathrm{l} / 00007038$ and $\mathrm{BBS} / \mathrm{E} / \mathrm{I} / 00007039$.

Acknowledgments: The authors would like to thank Prof Linda Saif, Ohio State University, for kindly providing porcine deltacoronavirus for use in this work. We would also like to thank Prof Miles Carroll, UK Health Security Agency, for kindly sharing SARS-CoV-2, hCov-19/England/02/2020.

Conflicts of Interest: The authors declare no conflicts of interest. The funders had no role in the design of the study; in the collection, analyses, or interpretation of data; in the writing of the manuscript, or in the decision to publish the results.

1. Ksiazek, T.G., Erdman, D. et al. A Novel Coronavirus Associated with Severe Acute

\section{References} 1953-1966.

2. Zaki, A.M., van Boheemen, S. et al. Isolation of a Novel Coronavirus from a Man with Pneumonia in Saudi Arabia. New England Journal of Medicine, 2012. 367(19): p. 1814-1820.

3. W.H.O. World Health Oganisation (WHO) Coronavirus (COVID-19) Dashboard With Vaccination Data. 2021 August 2021]; Available from: https://covid19.who.int.

4. Zhou, P., Yang, X.-L. et al. A pneumonia outbreak associated with a new coronavirus of probable bat origin. Nature, 2020. 579(7798): p. 270-273.

5. Miller, S. and Krijnse-Locker, J. Modification of intracellular membrane structures for virus replication. Nature Reviews Microbiology, 2008. 6(5): p. 363-374.

6. Nagy, P.D., Strating, J.R.P.M. et al. Building Viral Replication Organelles: Close

461 8. Snijder, E., Limpens, R.W.A.L. et al. A unifying structural and functional model of the coronavirus replication organelle: tracking down RNA synthesis. 2020, bioRxiv. 
463 9. Gosert, R., Kanjanahaluethai, A. et al. RNA Replication of Mouse Hepatitis Virus

464

465

466

467

468

469

470

471

472

473

474

475

476

477

478

479

480

481

482

483

484

485

486

487

488

489

490

491

492

493

494

495

496

497

498

499

500

501

502

503

504

505

Takes Place at Double-Membrane Vesicles. Journal of Virology, 2002. 76(8): p. 3697-3708.

10. Boon, J.A.d. and Ahlquist, P. Organelle-Like Membrane Compartmentalization of Positive-Strand RNA Virus Replication Factories. Annual Review of Microbiology, 2010. 64(1): p. 241-256.

11. Neufeldt, C.J., Joyce, M.A. et al. The Hepatitis C Virus-Induced Membranous Web and Associated Nuclear Transport Machinery Limit Access of Pattern Recognition Receptors to Viral Replication Sites. PLOS Pathogens, 2016. 12(2): p. e1005428.

12. Nagy, P.D. and Lin, W. Taking over Cellular Energy-Metabolism for TBSV Replication: The High ATP Requirement of an RNA Virus within the Viral Replication Organelle. Viruses, 2020. 12(1): p. 56.

13. Harak, C. and Lohmann, V. Ultrastructure of the replication sites of positive-strand RNA viruses. Virology, 2015. 479-480: p. 418-433.

14. Paul, D. and Bartenschlager, R. Architecture and biogenesis of plus-strand RNA virus replication factories. World J Virol, 2013. 2(2): p. 32-48.

15. Ávila-Pérez, G., Rejas, M.T. et al. Ultrastructural characterization of membranous torovirus replication factories. Cellular Microbiology, 2016. 18(12): p. 1691-1708.

16. Gosert, R., Egger, D. et al. Identification of the Hepatitis C Virus RNA Replication Complex in Huh-7 Cells Harboring Subgenomic Replicons. Journal of Virology, 2003. 77(9): p. 5487-5492.

17. Monaghan, P., Cook, H. et al. The ultrastructure of the developing replication site in foot-and-mouth disease virus-infected BHK-38 cells. Journal of General Virology, 2004. 85(4): p. 933-946.

18. Belov, G.A., Nair, V. et al. Complex Dynamic Development of Poliovirus Membranous Replication Complexes. Journal of Virology, 2012. 86(1): p. 302-312.

19. Limpens, R.W.A.L., Schaar, H.M.v.d. et al. The Transformation of Enterovirus Replication Structures: a Three-Dimensional Study of Single- and Double-Membrane Compartments. mBio, 2011. 2(5): p. e00166-00111.

20. Melia, C.E., Peddie, C.J. et al. Origins of Enterovirus Replication Organelles Established by Whole-Cell Electron Microscopy. mBio, 2019. 10(3): p. e00951-00919.

21. Cortese, M., Goellner, S. et al. Ultrastructural Characterization of Zika Virus Replication Factories. Cell Reports, 2017. 18(9): p. 2113-2123.

22. Gillespie, L.K., Hoenen, A. et al. The Endoplasmic Reticulum Provides the Membrane Platform for Biogenesis of the Flavivirus Replication Complex. Journal of Virology, 2010. 84(20): p. 10438-10447.

23. Offerdahl, D.K., Dorward, D.W. et al. A three-dimensional comparison of tick-borne flavivirus infection in mammalian and tick cell lines. PLoS One, 2012. 7(10): p. e47912.

24. Welsch, S., Miller, S. et al. Composition and Three-Dimensional Architecture of the Dengue Virus Replication and Assembly Sites. Cell Host \& Microbe, 2009. 5(4): p. 365-375. 
506

507

508

509

510

511

512

513

514

515

516

517

518

519

520

521

522

523

524

525

526

527

528

529

530

531

532

533

534

535

536

537

538

539

540

541

542

543

544

545

546

547

25. Schwartz, M., Chen, J. et al. Alternate, virus-induced membrane rearrangements support positive-strand RNA virus genome replication. Proceedings of the National Academy of Sciences of the United States of America, 2004. 101(31): p. 11263-11268.

26. Grimley, P.M., Berezesky, I.K. et al. Cytoplasmic structures associated with an arbovirus infection: loci of viral ribonucleic acid synthesis. J Virol, 1968. 2(11): p. 1326-1338.

27. Grimley, P.M., Levin, J.G. et al. Specific membranous structures associated with the replication of group A arboviruses. J Virol, 1972. 10(3): p. 492-503.

28. Kujala, P., Ikäheimonen, A. et al. Biogenesis of the Semliki Forest virus RNA replication complex. J Virol, 2001. 75(8): p. 3873-3884.

29. Miller, D.J., Schwartz, M.D. et al. Flock House Virus RNA Replicates on Outer Mitochondrial Membranes in Drosophila Cells. Journal of Virology, 2001. 75(23): p. 11664-11676.

30. Ertel, K.J., Benefield, D. et al. Cryo-electron tomography reveals novel features of a viral RNA replication compartment. eLife, 2017. 6: p. e25940.

31. Kopek, B.G., Perkins, G. et al. Three-dimensional analysis of a viral RNA replication complex reveals a virus-induced mini-organelle. PLoS Biol, 2007. 5(9): p. e220.

32. de Wilde, A.H., Raj, V.S. et al. MERS-coronavirus replication induces severe in vitro cytopathology and is strongly inhibited by cyclosporin A or interferon-a treatment. $J$ Gen Virol, 2013. 94(Pt 8): p. 1749-1760.

33. Goldsmith, C.S., Tatti, K.M. et al. Ultrastructural Characterization of SARS Coronavirus. Emerging Infectious Disease journal, 2004. 10(2): p. 320.

34. Snijder, E.J., van der Meer, Y. et al. Ultrastructure and Origin of Membrane Vesicles Associated with the Severe Acute Respiratory Syndrome Coronavirus Replication Complex. Journal of Virology, 2006. 80(12): p. 5927-5940.

35. Zhou, X., Cong, Y. et al. Ultrastructural Characterization of Membrane Rearrangements Induced by Porcine Epidemic Diarrhea Virus Infection. Viruses, 2017. 9(9): p. 251.

36. Doyle, N., Hawes, P.C. et al. The Porcine Deltacoronavirus Replication Organelle Comprises Double-Membrane Vesicles and Zippered Endoplasmic Reticulum with Double-Membrane Spherules. Viruses, 2019. 11(11): p. 1030.

37. Maier, H.J., Hawes, P.C. et al. Infectious Bronchitis Virus Generates Spherules from Zippered Endoplasmic Reticulum Membranes. mBio, 2013. 4(5).

38. van Hemert, M.J., van den Worm, S.H.E. et al. SARS-Coronavirus Replication/Transcription Complexes Are Membrane-Protected and Need a Host Factor for Activity In Vitro. PLOS Pathogens, 2008. 4(5): p. e1000054.

39. Knoops, K., Kikkert, M. et al. SARS-Coronavirus Replication Is Supported by a Reticulovesicular Network of Modified Endoplasmic Reticulum. PLOS Biology, 2008. 6(9): p. e226.

40. Klein, S., Cortese, M. et al. SARS-CoV-2 structure and replication characterized by in situ cryo-electron tomography. Nature Communications, 2020. 11(1): p. 5885. 
548 41. Gadlage, M.J., Sparks, J.S. et al. Murine Hepatitis Virus Nonstructural Protein 4 Regulates Virus-Induced Membrane Modifications and Replication Complex Function. Journal of Virology, 2010. 84(1): p. 280-290.

552

42. Meer, Y.v.d., Snijder, E.J. et al. Localization of Mouse Hepatitis Virus Nonstructural Proteins and RNA Synthesis Indicates a Role for Late Endosomes in Viral Replication. Journal of Virology, 1999. 73(9): p. 7641-7657.

554

43. Lundin, A., Dijkman, R. et al. Targeting Membrane-Bound Viral RNA Synthesis Reveals Potent Inhibition of Diverse Coronaviruses Including the Middle East Respiratory Syndrome Virus. PLoS Pathog, 2014. 10(5): p. e1004166.

558

44. Wolff, G., Limpens, R.W.A.L. et al. A molecular pore spans the double membrane of the coronavirus replication organelle. Science, 2020. 369(6509): p. 1395-1398.

45. Casais, R., Thiel, V. et al. Reverse Genetics System for the Avian Coronavirus Infectious Bronchitis Virus. Journal of Virology, 2001. 75(24): p. 12359-12369.

46. Hull, R.N., Cherry, W.R. et al. The origin and characteristics of a pig kidney cell 563 strain, LLC-PK. In Vitro, 1976. 12(10): p. 670-677.

564

47. Hu, H., Jung, K. et al. Isolation and characterization of porcine deltacoronavirus from 565 pigs with diarrhea in the United States. J Clin Microbiol, 2015. 53(5): p. 1537-1548.

48. Jung, K., Hu, H. et al. Pathogenicity of 2 porcine deltacoronavirus strains in gnotobiotic pigs. Emerg Infect Dis, 2015. 21(4): p. 650-654.

49. Hagemeijer, M.C., Vonk, A.M. et al. Visualizing Coronavirus RNA Synthesis in Time by Using Click Chemistry. Journal of Virology, 2012. 86(10): p. 5808-5816.

50. Hackbart, M., Deng, X. et al. Coronavirus endoribonuclease targets viral polyuridine sequences to evade activating host sensors. Proceedings of the National Academy of Sciences, 2020. 117(14): p. 8094-8103.

573

574

575

51. Kint, J., Fernandez-Gutierrez, M. et al. Activation of the Chicken Type I Interferon Response by Infectious Bronchitis Coronavirus. Journal of Virology, 2015. 89(2): p. 1156-1167.

53. Beachboard, D.C., Anderson-Daniels, J.M. et al. Mutations across Murine Hepatitis

54. Clementz, M.A., Kanjanahaluethai, A. et al. Mutation in murine coronavirus

52. Xu, L., Khadijah, S. et al. The Cellular RNA Helicase DDX1 Interacts with Coronavirus Nonstructural Protein 14 and Enhances Viral Replication. Journal of Virology, 2010. 84(17): p. 8571-8583.

55. Doyle, N., Neuman, B. et al. Infectious Bronchitis Virus Nonstructural Protein 4 Alone Induces Membrane Pairing. Viruses, 2018. 10(9): p. 477.

56. Hagemeijer, M.C., Monastyrska, I. et al. Membrane rearrangements mediated by coronavirus nonstructural proteins 3 and 4. Virology, 2014. 458-459: p. 125-135. viral RNA and other viral proteins. Virology, 2018. 513: p. 75-84. 
591 58. Athmer, J., Fehr, A.R. et al. In Situ Tagged nsp15 Reveals Interactions with 592 Coronavirus Replication/Transcription Complex-Associated Proteins. mBio, 2017. 593 8(1): p. e02320-02316.

594 59. Brockway, S.M., Lu, X.T. et al. Intracellular Localization and Protein Interactions of 595 the Gene 1 Protein p28 during Mouse Hepatitis Virus Replication. Journal of 596 Virology, 2004. 78(21): p. 11551-11562.

597 60. Subissi, L., Posthuma, C.C. et al. One severe acute respiratory syndrome 598 coronavirus protein complex integrates processive RNA polymerase and 599 exonuclease activities. Proceedings of the National Academy of Sciences, 2014. 600 111(37): p. E3900-E3909.

601

602 